\title{
Dircom, profesión y academia
}

\author{
Magdalena Mut CAMACHO \\ Universitat Jaume I de Castelló \\ magda.mut@com.uji.es
}

Recibido: $14 / 11 / 2012$

Aceptado: 23/01/2013

\section{Resumen}

La revisión de la figura profesional del dircom se halla en un momento trascendente, por un lado la nueva perspectiva de una empresa más unitaria y humana, por otro las exigencias sociales de un mundo más confiable, junto con la evolución del mundo académico para adaptarse al Espacio Europeo de Educación Superior, confluyen en la necesidad de definir a la figura con rigor y visión.

Por tanto, desde el mundo académico nos encontramos ante un momento muy interesante y fructífero, pues es época de meditar y llevar a la realidad lo que desde los diferentes ámbitos se exige que ha de ser un profesional dircom.

Palabras clave: Director de comunicación, Dircom, reputación, comunicación corporativa, valores intangibles

\section{Dircom, Profession and Academy}

\begin{abstract}
Abastract
The review of the professional figure dircom is in an important moment, first the new perspective of a unified and human company, on the other social demands of a more reliable, together with the evolution of the academic world to adapt to European area of higher education, converge on the need to define the figure with insight. Therefore, from the academic world, we are facing a very interesting and fruitful, it is time to meditate and bring it to reality from different fields are required to be a professional dircom.

Keywords: Director of Communication, Dircom, reputation, corporate communications, intangibles assets

\section{Referencia normalizada}

MUT CAMACHO, Magdalena (2013): “DIRCOM, profesión y academia”. Estudios sobre el mensaje periodístico. Vol. 19. Núm. especial abril, págs.: 887-896. Madrid, Servicio de Publicaciones de la Universidad Complutense.
\end{abstract}

Sumario: 1. Introducción. 2. El director de comunicación desde la academia. 3. El Director de comunicación desde la sociedad. 4. El Director de Comunicación desde la empresa. 5. Los contenidos de una asignatura sobre Dirección de Comunicación. 6. Referencias bibliográficas

\section{Introducción}

La revisión de la figura profesional del dircom se halla en un momento trascendente, por un lado la nueva perspectiva de una empresa más unitaria y humana, por otro las exigencias sociales de un mundo más confiable, junto con la evolución del mundo académico para adaptarse al Espacio Europeo de Educación Superior, confluyen en la necesidad de definir a la figura con rigor y visión.

Las titulaciones derivadas de la disciplina de comunicación están, a través de nuevos planes de estudios, modelando sus contenidos a las nuevas tendencias tanto las formales, que exige el nuevo andamiaje europeo, como las temáticas, que exige esta actualidad en la que nos hallamos inmersos.

Por tanto, desde el mundo académico nos encontramos ante un momento muy interesante y fructífero, pues es época de meditar y llevar a la realidad lo que desde los diferentes ámbitos se exige que ha de ser un profesional dircom. 
La realidad competitiva nos indica que la figura del profesional dircom inicia un desafío conceptual y afronta un importante momento de cambio en el que su papel adquiere aún más importancia en la estrategia de la organización. Ello es porque su vinculación con el entorno es sustancial y, además, la compresión de la existencia de una nueva visión de la empresa más unitaria, cercana y perceptiva, sirven para perfeccionar su vanguardia conceptual. Desde este punto de partida, el presente trabajo se encarga de analizar cuales deben ser los contenidos docentes que debe impartir una asignatura sobre la Dirección de Comunicación.

Arrancaremos desde el conocimiento de que el profesional encargado de la comunicación en las organizaciones se está viendo inmerso en un momento trascendental que nos obliga a los docentes a replantearnos cómo hemos de afrontar la enseñanza de las habilidades y las capacidades que los alumnos han de adquirir para llegar a ser el profesional que se demanda.

Si definimos al dircom como el encargado de guardar la reputación de la empresa y como el encargado de velar por la confianza de los públicos de interés, debemos atender tres frentes imprescindibles: el triunfo de las teorías sobre la reputación, la investigación social sobre la confianza y sus implicaciones, y el manejo útil de los indicadores de intangibles para la toma de decisiones estratégicas.

Desde el punto de vista de la gestión, han sido las Direcciones de Comunicación las que han ido asumiendo funciones con un esfuerzo de transformación continuo. Estas áreas de gestión, herederas de los departamentos de prensa, gabinetes de comunicación, etc. han evolucionado y han redefinido el rol de la comunicación, aunándolo a los intangibles estratégicos, cada vez más crecientes en importancia (Arthur Page Society, 2012, European Communication Monitor, 2012, DIRCOM, 2010, Mut Camacho, 2010, Villafañe, 2007).

Por tanto, se propone desde la visión de la actualidad una revisión de contenidos académicos sobre cómo ha de ser un Director de Comunicación.

\section{El director de comunicación desde la academia}

La figura del director de comunicación es bastante joven en el mundo empresarial, y aún más desde las nuevas teorías que están dándole una forma más precisa y omnicomprensiva de qué es la gestión de los valores de una organización, más allá de los financieros.

Es por esta razón de juventud que adolece de un claro entendimiento en la mayoría de las empresas sobre cual es su misión y, por tanto, de qué disciplina científica proviene este profesional.

En la actualidad, con la desaparición de las antiguas licenciaturas y el nacimiento de los nuevos grados, universidades como la Jaume I de Castellón ha incluido en sus planes docentes de sus tres titulaciones de comunicación, la asignatura obligatoria de Dirección de Comunicación. Una firme apuesta y una forma de mostrarle a la sociedad de forma clara, que dónde se forma a un Director de Comunicación es en las titulaciones de Publicidad y Relaciones Públicas, Periodismo y Comunicación Audiovisual. Además de que el Departamento de Ciencias de la Comunicación de la Universitat Jaume I imparte desde el curso 2007-08, unos estudios de postgrado adap- 
tados a las directrices europeas. El Máster en Nuevas Tendencias y Procesos de Innovación en Comunicación posee la especialidad profesional en "Dirección Estratégica de la Comunicación" donde se ofrecen las claves para afrontar la gestión de la reputación corporativa en empresas e instituciones públicas, con una visión avanzada y prospectiva de lo que ha de ser un dircom actual.

Aún podemos añadir un valor más, ya que la universidad pública siempre ha apostado por contenidos relativos a cuestiones que tienen que ver con la ética de las organizaciones y la confianza que éstas deben ganarse para estar legitimadas ante la sociedad. Cuestión que es importante remarcar, puesto que hoy en día es un debate el de la responsabilidad que las empresas tienen con todos sus públicos de interés, con la sociedad en particular. Desde la academia siempre se ha tenido claro que una empresa virtuosa es aquella que consigue estructurar su funcionamiento a partir de la reciprocidad, la cooperación y la confianza. Aquella que consigue integrar todos los beneficios de los grupos afectados y el consenso de todos ellos. Es decir, la organización que disponga de un alto capital económico y social (García Marzá, 2004, pág. 64). Y esta convicción se ha llevado a los contenidos académicos con una clara visión de cómo ha de ser una empresa de presente y de futuro.

\section{El Director de comunicación desde la sociedad}

En la actualidad asistimos a una globalización completa dónde los incidentes son locales a la vez que internacionales, afectando a todo el orbe, sobre todo los incidentes negativos. Todo ello es debido exclusivamente al trabajo de los medios de comunicación y al impacto de las nuevas tecnologías de la comunicación, que informan al público de lo sucedido en lugares que hace unos años consideraríamos remotos y hoy en día parecen estar al lado (Mut Camacho, 2012: 4). Estos incidentes que acaban vinculando a los gobiernos y a los ciudadanos también incumben a las empresas, porque una de sus obligaciones para una correcta gestión de su reputación es adelantarse a los contextos sociales, estar vigilante de ellos.

Internet ha transformado de un modo radical el mercado de la información, el proceso unidireccional antiguo y sin retorno de comunicación entre usuarios y medios ha quedado cancelado y superado. La comunicación ha cambiado de manera vertiginosa, modificando estructuras que parecían sólidas, abriéndose a la participación de la ciudadanía. "Lo que antes era jerarquía y unidireccionalidad, ahora se muestra abierto y bidireccional. Gran parte de culpa la tiene el fenómeno Internet. Desde que en los años setenta comenzase su andadura una nueva era ha comportado un escenario distinto y cambiante en el mundo de la comunicación. Internet ha revolucionado los cimientos establecidos pudiéndose augurar que estamos ante un cambio de paradigma que es aprovechado por el mundo empresarial" (Bernad et al., 2012: 1442), convirtiéndose en una herramienta de comunicación corporativa donde no sólo hablar, sino también escuchar.

Ya lo dijo McLuhan en su Understanding Media al hablar de la era mecánica: "Nos estamos acercando rápidamente a la fase final de las extensiones del hombre: la simulación tecnológica de la conciencia, por la cual los procesos creativos del conocimiento se extenderán, colectiva y corporativamente, al conjunto de la sociedad 
humana, de un modo muy parecido a como ya hemos extendido nuestros sentidos y nervios con los diversos medios de comunicación. [...] En la edad eléctrica en la que nuestro sistema nervioso se ha extendido tecnológicamente hasta implicarnos con toda la humanidad e incorporarla toda en nuestro interior, participamos necesaria y profundamente de las consecuencias de todos nuestros actos. Ya no es posible adoptar el distante y disociado papel del occidental alfabetizado".

El Teatro del Absurdo dramatiza este nuevo dilema del occidental, del hombre de acción que parece no estar implicado en la acción. Éste es el origen y el atractivo de los payasos de Samuel Beckett. Tras tres mil años de explosión especialista y de creciente especialización y alineación en las extensiones tecnológicas del cuerpo, nuestro mundo, en un drástico cambio de sentido, se ha vuelto agente de compresión. Eléctricamente contraído, el globo no es más que una aldea. La velocidad eléctrica con que se juntaron todas las funciones sociales y políticas en una implosión repentina ha elevado la conciencia humana a la responsabilidad en un grado intenso" (McLuhan, 1996: 320).

Por tanto el ámbito de la opinión pública ha cambiado porque el alcance de la información y la posibilidad de manifestación se han multiplicado.

Es por ello que desde hace unos veinte años a esta parte, la comunicación corporativa, en España, conceptualmente se ha ensanchado de forma asombrosa, tanto en su carácter formal y tecnológico, como en los discursos, en los mensajes y los vínculos relacionales que utiliza la empresa para conseguir ser legitimada por sus públicos. Más aún en España, cuando además se sucedieron una serie de importantes transformaciones que añadieron complejidad al escenario en el que interactuaban el emisor y el receptor de la comunicación corporativa: se entró en la Comunidad Económica Europea, se privatizaron las empresas públicas, llegaron las empresas extranjeras, la bonanza económica, las marcas españolas saltaron hacia el mercado internacional. Y más recientemente, el despertar del sentido social de responsabilidad aplicado a las empresas y la revolución tecnológica de la comunicación. Aunque puedan encontrarse antecedentes hacia finales de los setenta con la llegada de la democracia, no será hasta bien entrados los años ochenta y la década de los noventa cuando la gestión estratégica de la comunicación, a nivel formal, se transformó realmente en un vector de competitividad fundamental dentro de los nuevos escenarios organizacionales y se empezó a comprender como un proceso de gestión integral (Álvarez Novell et al., 2011). Un pionero en la profesión y también visionario teórico de la comunicación corporativa como Costa, ya a finales de los años setenta, avanzó que la comunicación debía ser integrada para ser efectiva (Costa, 2001).

De esta forma, encontramos que el primer director de comunicación se encargó de gestionar las relaciones de la organización con los medios de comunicación. Más tarde se vio que era positivo integrar las comunicaciones de la empresa y el nuevo rol del dircom pasó a ser comunicar la empresa con criterios unitarios. Eso ofrecía una imagen más fuerte, coherente y más competitiva. Pero con el paso de los años y de los acontecimientos aquello ya no sirve, ahora sabemos que lo que las organizaciones deben suscitar es confianza. De forma que entra en juego la reputación de la mano de cuestiones como la ética, las relaciones con el entorno, el medioambiente, la respon- 
sabilidad, las promesas. Toda esta complejidad plantea nuevos escenarios para las empresas, y los nuevos escenarios plantean nuevas necesidades en los públicos que han de ser conocidas y dominadas. El nuevo director o directora de comunicación ya no puede abordar la situación con la antigua herramienta de la integración de las comunicaciones, pues tan solo está centrada en mensajes, medios, canales, procesos y se deja de lado lo importante: que la reputación no es una cuestión únicamente de comunicación, también es de conducta. El dircom de hoy es un estratega de la comunicación y de la conducta para conseguir la confianza de sus colaboradores, de todos sus públicos de interés por el bien común de todos.

La empresa se manifiesta y es conocida, y en su grado máximo provoca simpatía, respeto y admiración, a través de tres discursos:

- Sus acciones.

- Su producto o servicio.

- Su comunicación.

Por lo tanto, ya no sirve un dircom que tan solo trabaje en el discurso de la comunicación. Su ámbito ha de abarcar los tres discursos, porque son estos tres los que construyen la reputación de la organización.

El dircom es el estratega de las cuestiones intangibles que agregan valor diferencial a la organización en estos tres discursos, ya no es el técnico que maneja tan solo el discurso de la comunicación, ahora tiene nuevas responsabilidades, justificadas por un nuevo escenario en el que hay que aprender a actuar de forma eficiente.

\section{El Director de Comunicación desde la empresa}

A estas alturas ya nadie duda ni discute la importancia y la presencia en cualquier actividad económica de la comunicación. Así como que la comunicación corporativa es una estrategia y no una herramienta de la organización.

La investigación El estado de la comunicación en España 2010 nos demuestra que el avance cualitativo del profesional es una realidad. A la pregunta sobre cómo ha evolucionado la gestión de la comunicación en los últimos cinco años la respuesta mayoritaria es para dos cuestiones intangibles estratégicas: la responsabilidad social $(68,59 \%)$ y la gestión de intangibles $(52,7 \%)$. Esta es una corriente actual pues a la pregunta sobre cuál será la tendencia evolutiva de la comunicación en los próximos años, la respuesta es un $81,82 \%$ es la responsabilidad social y un $78,15 \%$ es la gestión de intangibles.

Por otra parte, en el mismo estudio comprobamos como la incidencia en la estrategia de la empresa de la tarea estratégica del dircom es una evidencia constatable en la actualidad. 


\section{¿En qué medida contribuye la estrategia de Comunicación a la estrategia general de su empresa?}

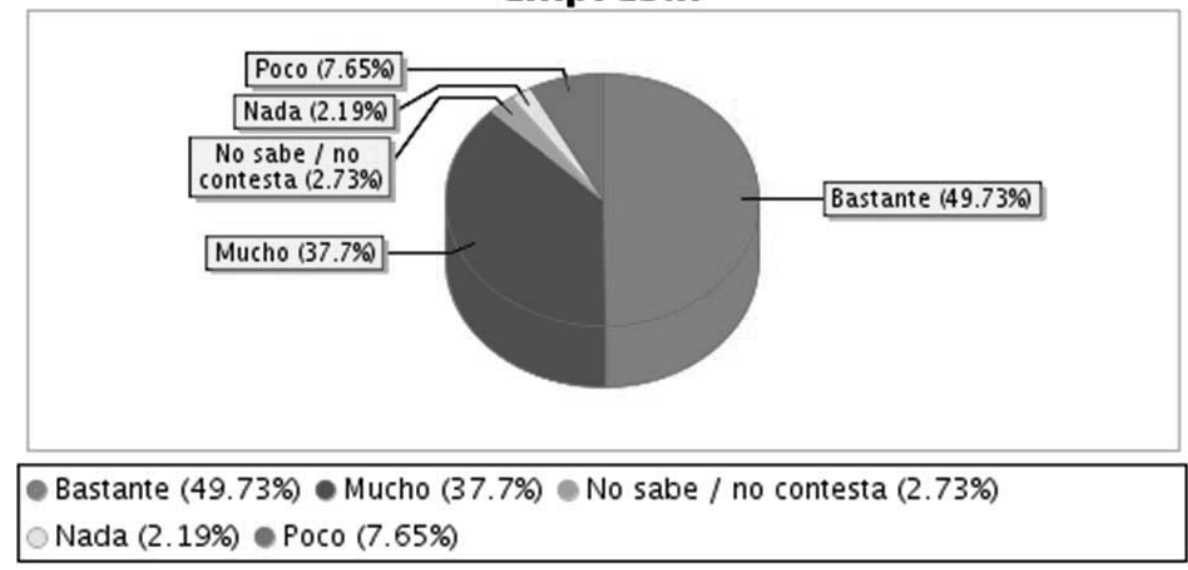

Fuente: Dircom

Entre la respuesta "bastante" y "mucho" suman un $87,43 \%$ de directores de comunicación que consideran que su tarea es estratégica. Por tanto, superado este paso categórico y condicional, hay que seguir avanzando.

Llegados a este punto es necesario recalcar que este camino intangible no es en contra de las gestiones tradicionales de la organización, es la culminación de una concepción de la empresa que lo engloba todo y le añade más valor.

El dircom de hoy conoce que la eficacia es la demanda fundamental de las empresas, que es necesario el avanzar en los modelos y metodologías de gestión de la reputación, y asumir como propias las tareas de la correcta gestión de los intangibles como adecuado proceder en este nuevo paradigma existente. Entiende que se ha modificado la conceptualización sobre la comunicación, a incluir la dimensión intangible de las organizaciones y a considerar la comunicación estratégica.

Hoy ya existen nuevos modelos de gestión que desplazan la práctica tradicional de la comunicación, tan polarizada en las técnicas y que ha descubierto la importancia decisiva de los activos intangibles, las relaciones, los valores y la reputación. Esta nueva concepción de lo que es la comunicación corporativa es el producto genérico y característico de nuestra época. Esta evolución es causa y efecto de la nueva forma de entender la empresa y su forma de comunicarse y relacionarse. Es un nuevo profesional.

\section{Los contenidos de una asignatura sobre Dirección de Comunicación}

A la luz de lo observado, consideramos que las capacidades y habilidades de un dircom han de ser las siguientes: 


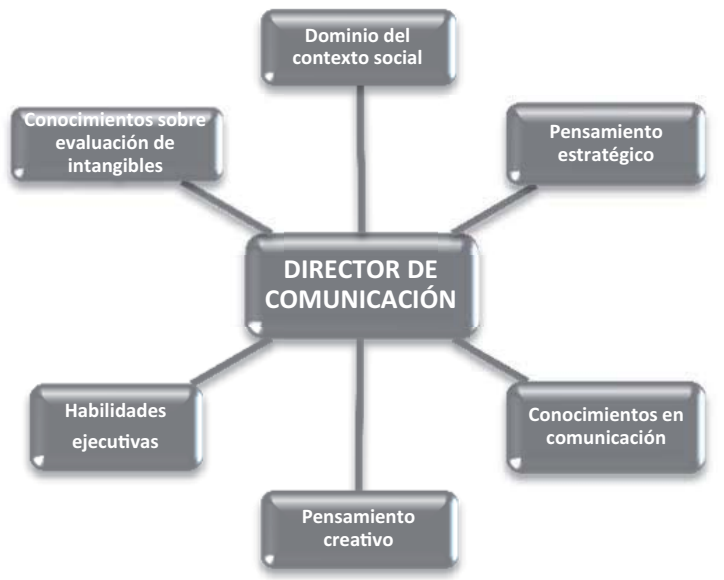

Fuente: elaboración propia

Sin olvidar el proceso evolutivo que ha llevado al dircom al lugar que ocupa conceptualmente hoy en día, y recordando el bagaje que le ha cimentado, "el primer paso en la construcción de la Dirección de Comunicación fue entregar la tarea de comunicación como parte de la estrategia empresarial, el siguiente paso, el que le dio cuerpo, fue convertirse ella misma en estrategia que añade valor a la estrategia de negocio y consolidar al Dircom como un estratega de la empresa, por último, la consolidación de la figura del Director de Comunicación pasa por la gestión de intangibles" (Mut Camacho, 2010: 29), se propone una figura encargada de la reputación de la organización.

Respecto a su formación, un enunciado rápido de los conocimientos imprescindibles que un Director de Comunicación debe dominar son: los correspondientes a la gestión empresarial, los que conciernen a la organización y a su cultura corporativa y, por último, los que atañen a la comunicación (Villafañe, 2001).

El carácter y originalidad del dircom se basa en esta fusión, en integrar los aspectos sobre el management con el conocimiento sobre su organización y con las teorías de la gestión intangible y la comunicación. Todo amalgamado con visión estratégica y pensamiento creativo. Planea y gestiona cuestiones cruciales de las que tan sólo él es especialista capacitado por su formación y su visión integradora y transversal de la acción estratégica.

Ciertamente la gestión de intangibles, como saber que bebe de muchas ciencias y saberes, conforma a un profesional con unos conocimientos y habilidades multidisciplinares. Se considera la formación universitaria en las disciplinas insertas en Ciencias de la Comunicación como las adecuadas, porque se hallan ubicadas en las Ciencias Sociales, área de conocimiento que hoy en día es primordial para la conformación de este moderno alto ejecutivo. A nivel teórico la comunicación se bifurca en dos grandes áreas: psicosociología y tecnología. "A la Dirección de Comunicación le interesará especialmente la primera, que es la que presenta déficit en las empresas. La segunda es cuestión de técnicos, y el Director de Comunicación no es un técnico 
ni un especialista, sino un estratega y un generalista polivalente. En sí misma, la ciencia de la comunicación resulta de la fusión de tres doctrinas: la Sistémica, la Teoría del feedback o retroacción y la teoría de la información. Esto es útil para entender la organización empresarial en tanto que un sistema vivo que interactúa consigo mismo y con su entorno socioeconómico y cultural" (Costa, 2001: 261). Es imprescindible el poseer los conocimientos y habilidades suficientes en el área específica de la gestión, análisis comercial y finanzas aplicadas, que le permitan participar de modo profundo en la toma de decisiones en la empresa, desde un prisma estratégico y gerencial (Garrido, 2004: 113).

Estos conocimientos reglados le servirán para ser ese profundo conocedor de la entidad para la cual presta sus servicios. Debe ser el experto en las relaciones de la organización, en las estrategias de intangibles y las tácticas de comunicación. Su actuación vendrá encaminada por una filosofía integradora en la que la obtención de beneficios es importante, pero también lo es el desarrollo común de los principios esenciales y cultura de la organización (Álvarez, 1997: 89; Martín Martín, 1995: 35; Villafañe, 1999: 100). Estas destrezas le otorgará el conocimiento real a través de los teóricos. Todo esto tiene el mérito de ubicarlo en la lógica empresarial, en el ámbito cotidiano donde tendrá que actuar.

Con su formación se complementan una serie de aptitudes como es la de permanecer atento al entorno, a lo que sucede en la sociedad, a cómo se desarrollan sus expectativas y saber traducirlas estratégicamente a los modos de la organización. Ello comporta mantener esa capacidad observadora, curiosa e investigadora de oportunidades. Es analítico con los datos que busca, desarrollando su capacidad intuitiva para comprender qué hay de beneficioso y que se puede hacer con ese mundo que evoluciona. Tendrá la visión estratégica para saber integrar la empresa en ese mundo vital y viceversa.

Una habilidad que se debe propiciar en el aula es la proactividad. Palabra procedente del latín, está compuesta por dos raíces latinas, pro-, que significa "ante" o "delante de", y -actividad, que significa "eficiencia de obrar, diligencia, eficacia", del latín activitas, -ātis. Viktor Frankl, un neurólogo y psiquiatra austriaco que sobrevivió a los campos de concentración nazis, introdujo este término en su libro de 1946 Man's Search for Meaning (El hombre en busca de sentido). Es una actitud en la que el sujeto u organización asume el pleno control de su conducta de modo activo, lo que implica la toma de iniciativa en el desarrollo de acciones creativas y audaces para generar mejoras, haciendo prevalecer la libertad de elección sobre las circunstancias del contexto.

En conclusión se propone la construcción de unos contenidos académicos que preparen al alumno para ser no únicamente un comunicador, o un experto en herramientas de comunicación, sino para ser el profesional que trabaja con expectativas, con tendencias, con hábitos y con emociones. Y todo dentro de un entorno global y complejo que debe conocer mejor que nadie en la empresa. El dircom es un generador de contenidos, debe conseguir que la realidad reputacional de la empresa sea similar a la percepción de los públicos. 


\section{Referencias bibliográficas}

ÁLVAREZ NOVELL, Alejandro y LESTA, Laura (2011): "Medición de los aportes de la gestión estratégica de comunicación interna a los objetivos de la organización". Palabra Clave. Vol. 14 (1), 11-30.

ÁLVAREZ, Tomás y CABALLERO, Mercedes (1997): Vendedores de imagen. Los retos de los nuevos gabinetes de comunicación. Barcelona, Paidós.

ARTHUR PAGE SOCIETY (2012): “Insights and Research”, en Arthur Page Society: http://www.awpagesociety.com/insights/building-belief/ [fecha de consulta: 1 de octubre de 2012]

BERNAD, Estela y MUT CAMACHO, Magdalena (2012): "Redes digitales y evento tradicional: Caso Festapedia". Revista de Comunicación Vivat Academia, pp. 14311444.

COSTA, Joan (2001): "El Director de Comunicación. La nueva figura central en la empresa del siglo XXI", en BENAVIDES, Juan: Dirección de Comunicación. Barcelona, Gestión 2000, pp. 47-66.

COSTA, Joan (2001): Imagen corporativa en el siglo XXI. Buenos Aires, La crujía.

DIRCOM (2010): Estado de la comunicación en España 2010. Madrid, DIRCOM.

EUROPEAN COMMUNICATION MONITOR (2012): Challenges and competencies for strategic communication, results of an empirical survey in 42 countries. EUPRERA.

GARCÍA MARZÁ, Domingo (2004): Ética empresarial: Del diálogo a la confianza. Madrid, Trotta.

GARCÍA-ALONSO, Pedro (2012): "Nuevos usuarios de la empresa informativa". Revista de Comunicación Vivat Academia, $n^{\circ}$ 117E, pp. 1391-1404.

GARRIDO, Francisco Javier (2004): Comunicación estratégica. Las claves de la comunicación empresarial en el siglo XXI. Barcelona, Gestión 2000.

MARTÍN MARTÍN, Fernando (1995): Comunicación en empresas e instituciones. De la consultora a la dirección de comunicación. Salamanca, Universidad de Salamanca.

MUT CAMACHO, Magdalena (2010): "Rumbo tras la la perspectiva comparada del director de comunicación". FISEC-Estrategias-Facultad de Ciencias Sociales de la Universidad Nacional de Lomas de Zamora. Año V, No 14, V4, pp. 25-39.

MUT CAMACHO, Magadalena (2012): "La opinión pública a pie de calle". Revista de Comunicación de la SEECI. (Julio 2012). Año XV (28), 1-10.

VILLAFAÑE, Justo (1999): La gestión profesional de la imagen corporativa. Madrid, Pirámide.

VILLAFAÑE, Justo (2001): "Prólogo". En VV.AA., Dirección de comunicación empresarial e institucional. Barcelona, Gestión 2000, pp. 14. 
VILLAFAÑE, Justo. (2007): "La gestión intangible”, en VILLAFAÑE, Justo: La comunicación empresarial y la gestión de los intengibles en España y Latinoamérica. Madrid, Pearson Prentice Hall, pp. 193-219.

\section{Magdalena MUT CAMACHO}

Universitat Jaume I de Castelló

Departament de Ciències de la Comunicació

Profesora contratada doctora

magda.mut@com.uji.es 